\title{
Joint Beamforming and the Artificial Noise Security Scheme Based on Successive Convex Approximation in Multi-relay Cognitive Network
}

\author{
Xian-zhong XIE ${ }^{1}$,Xiang-yu XIE ${ }^{1,2}$,Shu TANG ${ }^{1}$ and Jiu-jiu $\mathrm{CHEN}^{1}$ \\ ${ }^{1}$ Chongqing University of Posts and Telecommunications, China \\ ${ }^{2}$ xie_dave@qq.com
}

Keywords: Cognitive radio, Physical layer security, Multiple relay, Successive convex approximation, Joint optimization

\begin{abstract}
To solve the transmission problemof physical layer security(PLS) in relay cognitive radio network (CRN), this paper investigates the PLS transmission scheme of multiple relays CRN, which contains two pairs of secondary nodes without direct link each other. Compared with the model that consists ofa pair of secondary node users and a single relay in terms of theoretical analysis and optimization, the proposed scheme is more complicated. To maximizethe joint secret rate of the secondary users,power allocation of the twosecondarysource transmission node withbeamforming andartificial noise assist (PBNAS) is obtained. Furthermore, exploiting the semidefinite programming theory (SDR) and the successive convex approximation (SCA) method, the original complex nonconvex problem is transformed into a convex optimization problem, and the iterative procedure is summarized.Finally, the security transmission rate versus different number relays andrelays total power is simulated. The simulation results show that the security performance of the PBNAS scheme is the best.
\end{abstract}

\section{Introduction}

Cognitive radio technology aims at improving spectrum efficiency and alleviating spectrum resource scarcity. This technology allows non-authorized users (secondary users) to share legal frequency band with authorized users (primary users) as long as the former doesn't affect the later, thus it improving spectrum efficiency substantially. Nevertheless, the openness of the CRN structure makes it more likely to suffer the threat of security comparing to the traditional wireless network.[1,2] Under such circumstances, more attentions are paid to PLS technology for the sake of dealing with the security problems in the CRN. $[3,4,5]$ Relay CRN faces more security threats than ever. Bibliographies [4,5] summarized the process of researches in this field.

Nowadays, the researches of CRNPLS transmission scheme mainly focus on relay selection $[10,11,12,13,14]$ and relay beamforming [15,16]. There is also some research work reported in Chinese journals $[6,7,8,9]$. Given that relay beamforming is an important method of PLS, this paper mainly researches the CRNPLS transmission problems based on relay beamforming. There is a pair of nodes and some relay nodes in the bibliographies $[10,11,12,13,14]$, but they just select the best one of the relays to transmit signal. Although bibliographies $[15,16]$ mainly concern about CRNPLS transmission schemes with multi-relay collaboration, they concern only the condition of a pair of secondary users. The optimal aims of them are to receive the required signal to noise ratio (SNR), while sacrifice the secure transmission performance of the system.

In the CRN, because of the openness of the wireless network, the data is likely to 
be eavesdropped in the transmission, and because of the landform, the weather and so on, there is not direct link betweensourceand receiving node, so the transmission of relays collaboration is necessary. In fact, there are many cognitive nodes in the network that need to send informationat the same time, and relay nodes can't deal with the received information one by one. Therefore, it is necessary to study the security transmission scheme of multiple relays with beamforming and artificial noise under the constraint of the main user's interference temperature and to ensure the safe and effective transmission of the data. Based on the analysis above, one eavesdropper node, two pairs of secondary nodes and some relays are included in this paper and assumes all nodes in model with only one antenna. It aims at maximizing the joint secrecy rate of two secondary nodes. We notice that bibliographies $[17,18]$ study the optimization of secure rate under relay power constraint. This paper expands its research field in the condition of multiple relay $\mathrm{CRN}$, which makes theoretical analysis more complicated. Because the joint secrecy rate is a nonconvex problem, this paper combines (SDR) and (SCA) to transform the original nonconvex problem into the convex problem. Then reaching the process of iterative algorithm. In the end, after the stimulation of secrecy rate of different total power and number of relays, comes to the conclusion that the security performance of PBNAS in this paper is the best.

\section{System Model}

The model of CRN is displayed in Figure1. This system includes two secondary users' source nodes $\left(\mathrm{S}_{1}, \mathrm{~S}_{2}\right)$, some relays, two secondary users' destination nodes $\left(\mathrm{D}_{1}, \mathrm{D}_{2}\right)$ and one primary, as well as one eavesdropper.

Assuming that there is no direct link between secondary users' source nodes and primary, secondary users' destination nodes, that is to say, there doesn't exist the path $\mathrm{S}_{1}-\mathrm{P}, \mathrm{S}_{1}-\mathrm{R}, \mathrm{S}_{2}-\mathrm{P}, \mathrm{S}_{2}-\mathrm{R}$. Every node has only one antenna, and the noise value of every node is white Gaussian noise. The channel gain vector of $S_{1}-R, S_{1}-E, R-D_{1}$ is expressed as $g_{1}, \boldsymbol{e}_{1}, \boldsymbol{g}_{2}$, the channel gain vector of $\mathrm{S}_{2}-\mathrm{R}, \mathrm{S}_{2}-\mathrm{E}, \mathrm{R}-\mathrm{D}_{2}$ is expressed as $f_{1}, \boldsymbol{e}_{2}, \boldsymbol{f}_{2}$ and the channel gain vector of R-P is expressed ash.

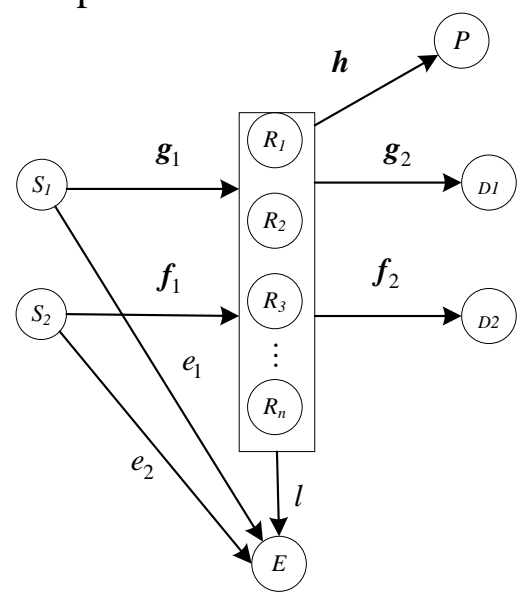

Figure 1. System model

We adopt the scheme of the two processes withrelaysamplify and forward combining beamforming and AN assist. In the first process, the two secondary source nodes broadcast messages. In the second process, relays adopt the method of beamforming transmitting the received message and AN. In both two processes, the eavesdroppercan eavesdrop the signals. This paper let $\mathrm{x}_{1}$ and $\mathrm{x}_{2}$ represent the desired emission signal of $S_{1}$ and $S_{2}$. Making $x_{1}$ and $x_{2}$ own unit power,without loss of generality $\mathrm{E}\left\{\left|\mathrm{x}_{1}\right|^{2}\right\}=\mathrm{E}\left\{\left|\mathrm{x}_{2}\right|^{2}\right\}=1$. 
The signal vector received by the relay in the first process:

$$
\boldsymbol{y}_{\boldsymbol{R}}=\sqrt{\mathrm{P}_{1}} \boldsymbol{g}_{1} \mathrm{x}_{1}+\sqrt{\mathrm{P}_{2}} \boldsymbol{f}_{\mathbf{1}} \mathrm{x}_{2}+\boldsymbol{n}_{\boldsymbol{1}} \text {. }
$$

$\boldsymbol{y}_{\mathrm{R}}=\left[y_{\mathrm{R}_{1}}, y_{\mathrm{R}_{2}}, \cdots, y_{\mathrm{R}_{\mathrm{N}}}\right]^{\mathrm{H}}, \quad \boldsymbol{y}_{\mathrm{R}_{\mathrm{N}}}$ means the received signals of the $\mathrm{Nth}$ relay node. $P_{1}$, $P_{2}$ means the transmission power of the two secondary nodes. $P_{A}$ denotes the total power of transmitting nodes. $\alpha$ is the allocation coefficient. $\alpha \in[0,1]$, in fact $P_{1}=\alpha P_{A}, P_{2}=(1-\alpha) P_{A}$

The signal vector received by the eavesdrop nodes in the first process:

$$
\mathrm{y}_{\mathrm{E} 1}=\sqrt{\mathrm{P}_{1}} \boldsymbol{e}_{1} \mathrm{x}_{1}+\sqrt{\mathrm{P}_{2}} \boldsymbol{e}_{2} \mathrm{x}_{2}+\boldsymbol{n}_{e l} .
$$

In the second period, relays amplify and forward the received signals and transmit the ANto disordereavesdropper. Let $\boldsymbol{a}$ and $\boldsymbol{b}$ be one-dimensional column $\operatorname{vector}(\mathrm{N} \times 1)$. Because of $\operatorname{diag}(\boldsymbol{b}) \boldsymbol{a}=\operatorname{diag}(\boldsymbol{a}) \boldsymbol{b}$, we can get the relay transmitting signal:

$$
\begin{aligned}
& \boldsymbol{x}_{\boldsymbol{R}}=\overline{\boldsymbol{W}}\left(\sqrt{\mathrm{P}_{1}} \boldsymbol{g}_{\boldsymbol{l}} \mathrm{x}_{1}+\sqrt{\mathrm{P}_{2}} \boldsymbol{f}_{\boldsymbol{l}} \mathrm{x}_{2}+\mathrm{n}_{1}\right)+\boldsymbol{z} \\
& =\sqrt{\mathrm{P}_{1}} \operatorname{diag}\left(\boldsymbol{g}_{\boldsymbol{l}}\right) \boldsymbol{w} \mathrm{x}_{1}+\sqrt{\mathrm{P}_{2}} \operatorname{diag}\left(\boldsymbol{f}_{\boldsymbol{l}}\right) \boldsymbol{w} \mathrm{x}_{2}+\overline{\boldsymbol{W}} \mathrm{n}_{1}+\boldsymbol{z} .
\end{aligned}
$$

In the equation, $\overline{\boldsymbol{W}}=\operatorname{diag}(\boldsymbol{w}), \boldsymbol{w}=\left[\mathrm{w}_{1}, \mathrm{w}_{2}, \cdots, \mathrm{w}_{\mathrm{N}}\right]^{\mathrm{H}}$ is the vector of beamforming, and $z$ is the vector of the ANthat relays transmit, which follows the zero mean Gaussian distribution, that is, $z \sim C N(0, \Sigma), \Sigma$ is the covariance matrixof the $\mathrm{AN} z$. $\Sigma=\mathrm{E}\left\{[z-\mathrm{E}(z)]\left[z-\mathrm{E}(z)^{\mathrm{H}}\right]\right\}=\mathrm{E}\left\{z z^{\mathrm{H}}\right\}, \quad \Sigma \succeq 0$ means that $\Sigma$ is Hermitian Conjugate Matrix,so the total power consumption of relays:

$$
\begin{aligned}
& \mathrm{P}_{\mathrm{R}}=\operatorname{Tr}\left(\mathrm{P}_{1} \operatorname{diag}\left(\boldsymbol{g}_{\boldsymbol{l}}\right) \boldsymbol{W} \operatorname{diag}\left(\boldsymbol{g}_{\boldsymbol{l}}\right)\right)+\operatorname{Tr}\left(\boldsymbol{z} \boldsymbol{z}^{\mathrm{H}}\right)+ \\
& \operatorname{Tr}\left(\mathrm{P}_{2} \operatorname{diag}\left(\boldsymbol{f}_{\boldsymbol{l}}\right) \boldsymbol{W} \operatorname{diag}\left(\boldsymbol{f}_{\boldsymbol{l}}\right)\right)+\operatorname{Tr}(\boldsymbol{W}) \sigma_{1}^{2} .
\end{aligned}
$$

In the expression, $\boldsymbol{W}=\boldsymbol{w} \boldsymbol{w}^{\mathrm{H}}$ and $\operatorname{Tr}$ means the trace of the matrix.

The signal transmitted by relays and received by primary user is $\mathrm{y}_{\mathrm{P}}=\boldsymbol{h}^{\mathrm{H}} \boldsymbol{x}_{\mathrm{R}}$. Making $\boldsymbol{h}_{g_{I}}^{\mathrm{H}}=\boldsymbol{h}^{\mathrm{H}} \operatorname{diag}\left(\boldsymbol{g}_{\boldsymbol{l}}\right), \boldsymbol{h}_{\boldsymbol{f l}}^{\mathrm{H}}=\boldsymbol{h}^{\mathrm{H}} \operatorname{diag}\left(\boldsymbol{f}_{\boldsymbol{l}}\right)$, and because of $\operatorname{diag}(\operatorname{diag}(\boldsymbol{W}))=\overline{\boldsymbol{W}} \overline{\boldsymbol{W}}$, thus the interference temperature that the primary usersuffers:

$$
\mathrm{I}_{\mathrm{P}}=\mathrm{P}_{1} \boldsymbol{h}_{\boldsymbol{g} \boldsymbol{I}}^{\mathrm{H}} \boldsymbol{W} \boldsymbol{h}_{\boldsymbol{g} \boldsymbol{I}}+\mathrm{P}_{2} \boldsymbol{h}_{\boldsymbol{f} \boldsymbol{H}}^{\mathrm{H}} \boldsymbol{W} \boldsymbol{h}_{\boldsymbol{f} \boldsymbol{l}}+\sigma_{\mathrm{r}}^{2} \boldsymbol{h}^{\mathrm{H}} \operatorname{diag}(\operatorname{diag}(\boldsymbol{W})) \boldsymbol{h}+\operatorname{Tr}\left(\boldsymbol{h}^{\mathrm{H}} \boldsymbol{\Sigma} \boldsymbol{h}\right) .
$$

The signals that the first and second node receives:

$$
y_{\mathrm{D}_{1}}=\boldsymbol{g}_{2}{ }^{\mathrm{H}} x_{R}+n_{r 1}, y_{\mathrm{D}_{2}}=\boldsymbol{f}_{2}{ }^{\mathrm{H}} x_{R}+n_{r 2} \text {. }
$$

The signal that the eavesd ropper receives in the second process:

$$
\mathrm{y}_{\mathrm{E}_{2}}=\boldsymbol{l}^{\mathrm{H}} \mathrm{x}_{\mathrm{R}}+\mathrm{n}_{\mathrm{e} 2} \text {. }
$$

\section{Joint Beamforming and AN}

Bring (3)in (6). To simplify the equation, making $\boldsymbol{g}_{2 g_{1}}^{\mathrm{H}}=\boldsymbol{g}_{2}{ }^{\mathrm{H}} \operatorname{diag}\left(\boldsymbol{g}_{\boldsymbol{1}}\right), \boldsymbol{g}_{2 f_{1}}^{\mathrm{H}}=\boldsymbol{g}_{2}{ }^{\mathrm{H}} \operatorname{diag}\left(\boldsymbol{f}_{\boldsymbol{1}}\right)$, $\boldsymbol{f}_{{ }_{2 g_{I}}}^{\mathrm{H}}=\boldsymbol{f}_{2}{ }^{\mathrm{H}} \operatorname{diag}\left(\boldsymbol{g}_{\boldsymbol{l}}\right), \boldsymbol{f}_{2 f_{l}}^{\mathrm{H}}=\boldsymbol{f}_{2}{ }^{\mathrm{H}} \operatorname{diag}\left(\boldsymbol{f}_{\boldsymbol{l}}\right), \boldsymbol{l}_{g_{I}}^{\mathrm{H}}=\boldsymbol{l}^{\mathrm{H}} \operatorname{diag}\left(\boldsymbol{g}_{\boldsymbol{l}}\right), \boldsymbol{l}_{f_{l}}^{\mathrm{H}}=\boldsymbol{l}^{\mathrm{H}} \operatorname{diag}\left(\boldsymbol{f}_{\boldsymbol{l}}\right)$. We can get the SNR of twosecondary destination nodes: 


$$
\begin{aligned}
& \gamma_{\mathrm{D}_{1}}=\frac{\mathrm{P}_{1} \boldsymbol{g}_{2 g_{1}}^{\mathrm{H}} \boldsymbol{W} \boldsymbol{g}_{2 g_{1}}}{\mathrm{P}_{2} \boldsymbol{g}_{2 f_{1}}^{\mathrm{H}} \boldsymbol{W} \boldsymbol{g}_{2 f_{1}}+\boldsymbol{g}_{2}^{\mathrm{H}} \boldsymbol{\Sigma} \boldsymbol{g}_{2}+\sigma_{\mathrm{r} 1}^{2}+\sigma_{\mathrm{r}}^{2} \boldsymbol{g}_{2}^{\mathrm{H}} \operatorname{diag}(\operatorname{diag}(\boldsymbol{W})) \boldsymbol{g}_{2}} . \\
& \gamma_{\mathrm{D}_{2}}=\frac{\mathrm{P}_{2} \boldsymbol{f}_{2 f_{1}}^{\mathrm{H}} \boldsymbol{W} \boldsymbol{f}_{2 f_{1}}}{\mathrm{P}_{1} \boldsymbol{f}_{2 g_{1}}^{\mathrm{H}} \boldsymbol{W} \boldsymbol{f}_{2 g_{1}}+\boldsymbol{f}_{2}^{\mathrm{H}} \boldsymbol{\Sigma} \boldsymbol{f}_{2}+\sigma_{\mathrm{r} 2}^{2}+\sigma_{\mathrm{r}}^{2} \boldsymbol{f}_{2}^{\mathrm{H}} \operatorname{diag}(\operatorname{diag}(\boldsymbol{W})) \boldsymbol{f}_{2}} .
\end{aligned}
$$

From (2) can get, the SNR of eavesdropperin the first process:

$$
\gamma_{\mathrm{E}_{11}}=\frac{\mathrm{P}_{1} \boldsymbol{e}_{1}^{2}}{\mathrm{P}_{2} \boldsymbol{e}_{2}^{2}+\sigma_{\mathrm{e}}^{2}}, \quad \gamma_{\mathrm{E}_{12}}=\frac{\mathrm{P}_{2} \boldsymbol{e}_{2}^{2}}{\mathrm{P}_{1} \boldsymbol{e}_{1}^{2}+\sigma_{\mathrm{e}}^{2}} .
$$

From (7) can get, the SNR of eavesdropperin the second process:

$$
\begin{aligned}
& \gamma_{\mathrm{E}_{21}}=\frac{\mathrm{P}_{1} \boldsymbol{l}_{g_{l}}^{\mathrm{H}} \boldsymbol{W} \boldsymbol{l}_{g_{l}}}{\mathrm{P}_{2} \boldsymbol{l}_{f_{l}}^{\mathrm{H}} \boldsymbol{W} \boldsymbol{l}_{f_{1}}+\boldsymbol{l}^{\mathrm{H}} \boldsymbol{\Sigma} \boldsymbol{l}+\sigma_{\mathrm{e} 2}^{2}+\sigma_{\mathrm{r}}^{2} \boldsymbol{l}^{\mathrm{H}} \operatorname{diag}(\operatorname{diag}(\boldsymbol{W})) \boldsymbol{l}} . \\
& \gamma_{\mathrm{E}_{22}}=\frac{\mathrm{P}_{2} \boldsymbol{l}_{f_{\boldsymbol{l}}}^{\mathrm{H}} \boldsymbol{W} \boldsymbol{l}_{f_{\boldsymbol{l}}}}{\mathrm{P}_{\boldsymbol{l}_{g_{I}}}^{\mathrm{H}} \boldsymbol{W} \boldsymbol{l}_{g_{1}}+\boldsymbol{l}^{\mathrm{H}} \boldsymbol{\Sigma} \boldsymbol{l}+\sigma_{\mathrm{e} 2}^{2}+\sigma_{\mathrm{r}}^{\boldsymbol{l}^{\mathrm{H}}} \operatorname{diag}(\operatorname{diag}(\boldsymbol{W})) \boldsymbol{l}} .
\end{aligned}
$$

Adoptingmaximum ratio combining,so secrecy rate of two secondary nodes:

$$
\begin{aligned}
& \mathrm{R}_{\mathrm{S}_{1}}(\boldsymbol{W}, \Sigma)=\mathrm{R}_{\mathrm{D}_{1}}-\mathrm{R}_{\mathrm{E}_{1}}=\frac{1}{2} \log _{2}\left(1+\gamma_{\mathrm{D}_{1}}\right)-\frac{1}{2} \log _{2}\left(1+\gamma_{\mathrm{E}_{11}}+\gamma_{\mathrm{E}_{21}}\right) . \\
& \mathrm{R}_{\mathrm{S}_{2}}(\boldsymbol{W}, \Sigma)=\mathrm{R}_{\mathrm{D}_{2}}-\mathrm{R}_{\mathrm{E}_{2}}=\frac{1}{2} \log _{2}\left(1+\gamma_{\mathrm{D}_{2}}\right)-\frac{1}{2} \log _{2}\left(1+\gamma_{\mathrm{E}_{21}}+\gamma_{\mathrm{E}_{22}}\right) .
\end{aligned}
$$

Let $\mathrm{A}=\mathrm{P}_{2} \boldsymbol{g}_{2 f_{1}}^{\mathrm{H}} \mathrm{W} \boldsymbol{g}_{2 f_{1}}+\sigma_{\mathrm{r}}^{2} \boldsymbol{g}_{2}^{\mathrm{H}} \operatorname{diag}(\operatorname{diag}(\boldsymbol{W})) \boldsymbol{g}_{2}+\boldsymbol{g}_{2}^{\mathrm{H}} \boldsymbol{\Sigma} \boldsymbol{g}_{2}+\sigma_{\mathrm{r} 1}^{2} \quad, \quad \mathrm{~B}=\mathrm{P}_{2} \boldsymbol{e}_{2}^{2}+\sigma_{\mathrm{e}}^{2}$, $\mathrm{C}=\mathrm{P}_{1} \boldsymbol{l}_{g_{1}}^{\mathrm{H}} \boldsymbol{W} \boldsymbol{l}_{g_{1}}, \quad \mathrm{D}=\mathrm{P}_{2} \boldsymbol{l}_{f_{\boldsymbol{l}}}^{\mathrm{H}} \boldsymbol{W} \boldsymbol{l}_{f_{\boldsymbol{I}}}+\sigma_{\mathrm{r}}^{2} \boldsymbol{l}^{\mathrm{H}} \operatorname{diag}(\operatorname{diag}(\boldsymbol{W})) \boldsymbol{l}+\boldsymbol{l}^{\mathrm{H}} \boldsymbol{\Sigma} \boldsymbol{l}+\sigma_{\mathrm{e} 2}^{2}, \mathrm{C}_{1}=\mathrm{P}_{2} \boldsymbol{l}_{f_{\boldsymbol{l}}}^{\mathrm{H}} \boldsymbol{W} \boldsymbol{l}_{f_{\boldsymbol{l}}}$ $\mathrm{A}_{1}=\mathrm{P}_{1} \boldsymbol{f}_{2 g_{1}}^{\mathrm{H}} \boldsymbol{W} \boldsymbol{f}_{2 g_{1}}+\sigma_{\mathrm{r}}^{2} \boldsymbol{f}_{2}^{\mathrm{H}} \operatorname{diag}(\operatorname{diag}(\boldsymbol{W})) \boldsymbol{f}_{2}+\boldsymbol{f}_{2}^{\mathrm{H}} \boldsymbol{\Sigma} \boldsymbol{f}_{2}+\sigma_{\mathrm{r} 2}^{2} \quad, \quad \mathrm{~B}_{1}=\mathrm{P}_{1} \boldsymbol{e}_{\boldsymbol{l}}^{2}+\sigma_{\mathrm{e}}^{2}$ $\mathrm{D}_{1}=\mathrm{P}_{1} \boldsymbol{l}_{g_{1}}^{\mathrm{H}} \boldsymbol{W} \boldsymbol{l}_{g_{1}}+\boldsymbol{l}^{\mathrm{H}} \boldsymbol{\Sigma} \boldsymbol{l}+\sigma_{\mathrm{e} 2}^{2}+\sigma_{\mathrm{r}}^{2} \boldsymbol{l}^{\mathrm{H}} \operatorname{diag}(\operatorname{diag}(\boldsymbol{W})) \boldsymbol{l}$, then get secrecy rate:

$$
\begin{gathered}
\mathrm{R}_{\mathrm{S}_{1}}(\boldsymbol{W}, \Sigma)=\frac{1}{2} \log _{2}\left(\frac{\mathrm{P}_{1} \boldsymbol{g}_{2 g_{1}}^{\mathrm{H}} \boldsymbol{W} \boldsymbol{g}_{2 g_{1}}+\mathrm{A}}{\mathrm{A}}\right)-\frac{1}{2} \log _{2}\left(\frac{\mathrm{P}_{1} \mathrm{e}_{1}^{2} \mathrm{D}+\mathrm{CB}+\mathrm{BD}}{\mathrm{BD}}\right) . \\
\mathrm{R}_{\mathrm{s}_{2}}(\boldsymbol{W}, \Sigma)=\frac{1}{2} \log _{2}\left(\frac{\mathrm{P}_{2} \boldsymbol{f}_{2 f_{1}}^{\mathrm{H}} \boldsymbol{W} \boldsymbol{f}_{2 f_{1}}+\mathrm{A}_{1}}{\mathrm{~A}_{1}}\right)-\frac{1}{2} \log _{2}\left(\frac{\mathrm{P}_{2} \mathrm{e}_{2}^{2} \mathrm{D}_{1}+\mathrm{C}_{1} \mathrm{~B}_{1}+\mathrm{B}_{1} \mathrm{D}_{1}}{\mathrm{~B}_{1} \mathrm{D}_{1}}\right) .
\end{gathered}
$$

Scalar coefficient $1 / 2$ is gained because when relay mode transmission is adopted, the source and the relay use half of time.

The joint secrecy rate is $R_{S_{1}}+R_{S_{2}}$, the interference temperature is $I_{\max }$ and the total powerlimit is $P_{\max }$, our optimization goal can be expressed as follows:

$$
\max _{(W, \Sigma)}\left(R_{\mathrm{S} 1}+R_{\mathrm{S} 2}\right)
$$

S.t. 


$$
P_{\max } \geq P_{\mathrm{R}}, I_{\max } \geq I_{\mathrm{P}}, \boldsymbol{W} \succeq 0 . \Sigma \succeq 0, \operatorname{rank}(\boldsymbol{W})=1 .
$$

The above formula is a function of beamforming weighting matrix $\boldsymbol{W}$ and theANcovariance matrix $\Sigma$. Under the condition that the total power of the source node $P_{A}$, the total power of the relay node $P_{\max }$ and the interference temperature of the primary user $I_{\max }$ is fixed, the secrecy rate cangrow to the maximum by jointly optimizing $\boldsymbol{W}$ and $\Sigma$. Since the original objective function is a complex nonconvex function, the solution is quite complex. So we use the SCA and SDR to get the optimal solution.

\section{The Joint Beamforming and the AN scheme of the SCA}

From above expression one can get:

$$
\max _{(W, \Sigma)}\left(\begin{array}{l}
\frac{1}{2}\left(\log _{2}\left(\frac{P_{1} \boldsymbol{g}_{2 g_{1}}^{\mathrm{H}} \boldsymbol{W} \boldsymbol{g}_{2 g_{1}}+A}{A}\right)-\log _{2}\left(\frac{P_{1} e_{1}^{2} D+C B+B D}{B D}\right)\right) \\
+\frac{1}{2}\left(\log _{2}\left(\frac{P_{2} \boldsymbol{f}_{2 f_{1}}^{\mathrm{H}} \boldsymbol{W} \boldsymbol{f}_{2 f_{1}}+A_{1}}{A_{1}}\right)-\log _{2}\left(\frac{P_{2} e_{2}^{2} D_{1}+C_{1} B_{1}+B_{1} D_{1}}{B_{1} D_{1}}\right)\right)
\end{array}\right)
$$

S.t.

$$
P_{\max } \geq P_{R}, I_{\max } \geq I_{P}, \boldsymbol{W} \succeq 0, \Sigma \succeq 0 .
$$

$\operatorname{rank}(\boldsymbol{W})=1$.

Firstly, SDR[19,21] is used to eliminate the nonconvex constraint of rank $1(18 \mathrm{~b})$. However, the objective function is still a complex nonconvex function, then we introduce the exponential function $\mathrm{e}^{x}$.

$$
\begin{aligned}
e^{x_{1}} & =P_{1} \boldsymbol{g}_{2 g_{1}}^{H} \boldsymbol{W g}{ }_{2 g_{1}}+A . \\
e^{x_{2}} & =A . \\
e^{x_{3}} & =P_{1} e_{1}^{2} D+C B+B D . \\
e^{x_{4}} & =B D . \\
e^{y_{1}} & =P_{2} f_{2 f_{1}}^{H} \boldsymbol{W} f_{2 f_{1}}+A_{1} . \\
e^{y_{2}} & =A_{1} . \\
e^{y_{3}} & =P_{2} e_{2}^{2} D_{1}+C_{1} B_{1}+B_{1} D_{1} . \\
e^{y_{4}} & =B_{1} D_{1} .
\end{aligned}
$$

Because of the $\left|\boldsymbol{g}^{\mathrm{H}} \boldsymbol{w}\right|^{2} \geq 0$, we can get: 


$$
\begin{aligned}
& e^{x_{1}} \geq \sigma_{r 1}^{2}, e^{x_{2}} \geq \sigma_{r 1}^{2} . \\
& e^{x_{3}} \geq P_{1} e_{1}^{2} \sigma_{e 2}^{2}+\left(P_{2} e_{2}^{2}+\sigma_{e}^{2}\right) \sigma_{e 2}^{2} . \\
& e^{x_{4}} \geq\left(P_{2} \mathbf{e}_{2}^{2}+\sigma_{\mathrm{e}}^{2}\right) \sigma_{\mathrm{e} 2}^{2} .
\end{aligned}
$$

The total power of the system is constrained, therefore $e^{x_{1}}, e^{x_{2}}, e^{x_{3}}, e^{x_{4}}, e^{y_{1}}, e^{y_{2}}, e^{y_{3}}, e^{y_{4}}$ will not be infiniteso $x_{1}, x_{2}, x_{3}, x_{4}, y_{1}, y_{2}, y_{3}, y_{4}$ are bounded. In order to replace the nonconvex expression (18) with the expression in (19), we do as following

$$
\max _{\left(W, \Sigma, x_{i}, y_{i} ; \in \in(1,2,3,4)\right)}\left(\frac{1}{2}\left(\left(x_{1}-x_{2}-x_{3}+x_{4}\right)+\left(y_{1}-y_{2}-y_{3}+y_{4}\right)\right) \log _{2}(\mathrm{e})\right)
$$

S.t.

(18a)

$$
P_{1} \boldsymbol{g}_{2 g_{1}}^{H} \boldsymbol{W} \boldsymbol{g}_{2 g_{1}}+A \geq e^{x_{1}}
$$

$A \leq e^{x_{2}}$.

$$
P_{l} e_{1}^{2} D+C B+B D \leq e^{x_{3}}
$$

$B D \geq e^{x_{4}}$.

$$
P_{2} \boldsymbol{f}_{2 f_{1}}^{H} \boldsymbol{W} \boldsymbol{f}_{2 f_{1}}+A_{1} \geq e^{y_{1}}
$$

$$
\begin{aligned}
& A_{1} \leq e^{y_{2}} . \\
& e^{y_{3}} \geq P_{2} e_{2}^{2} D_{1}+C_{1} B_{1}+B_{1} D_{1} . \\
& e^{y_{4}} \leq B_{1} D_{1} .
\end{aligned}
$$

As shown in (21), theoriginal objective function has been transformed into a convex function, and the equation in (19) has been replaced by inequalities (21b) to (21i). It can be verified by the monotonicity of the objective function that optimal values will be obtaineduntilall the equations from(21b) to (21i) hold.

$A, A_{1}, C, C_{1}, D, D_{1}$ contain $\boldsymbol{W}, \boldsymbol{\Sigma}$. We can adjust the value of $A, A_{1}, C, C_{1}, D, D_{1}$ acquiring the suitable $x_{1}, x_{2}, x_{3}, x_{4}, y_{1}, y_{2}, y_{3}, y_{4}$ Depending on the value abovethe maximum value of objective function can be obtained

But the constraint condition of (21c), (21d), (21g) (24h) are stillnonconvex. In order to convert these nonconvex constraints into convex constraints, let $\tilde{W}, \tilde{\Sigma}$ be thefeasible solution in (21). Using $\tilde{\boldsymbol{W}}, \tilde{\Sigma}$ replace $\boldsymbol{W}, \Sigma$ in $A, C, D, A_{1}, C_{1}, D_{1}$, so we can get $\tilde{A}, \tilde{C}, \tilde{D}, \tilde{A}_{1}, \tilde{C}_{1}, \tilde{D}_{1}$, then the expression in (19) is replaced by below:

$$
\tilde{x}_{2} \triangleq \ln (\tilde{A})
$$




$$
\begin{aligned}
& \tilde{x}_{3} \triangleq \ln \left(P_{1} e_{1}^{2} \tilde{D}+\tilde{C} B+B \tilde{D}\right) . \\
& \tilde{y}_{2} \triangleq \ln \left(\tilde{A}_{1}\right) . \\
& \tilde{y}_{3} \triangleq \ln \left(P_{l} e_{1}^{2} \tilde{D}_{1}+\tilde{C}_{1} B_{1}+B_{1} \tilde{D}_{1}\right) .
\end{aligned}
$$

The nonconvex constraint in (21) is extended with a first-order Taylor series ${ }^{[20]}$

$$
\begin{aligned}
& A \leq e^{\tilde{x}_{2}}\left(x_{2}-\tilde{x}_{2}+1\right) . \\
& P_{1} e_{1}^{2} D+C B+B D \leq e^{\tilde{x}_{3}}\left(x_{3}-\tilde{x}_{3}+1\right) . \\
& A_{1} \leq e^{\tilde{y}_{2}}\left(y_{2}-\tilde{y}_{2}+1\right) . \\
& P_{2} e_{2}^{2} D_{1}+C_{1} B_{1}+B_{1} D_{1} \leq e^{\tilde{y}_{3}}\left(y_{3}-\tilde{y}_{3}+1\right) .
\end{aligned}
$$

All of expressionin (23)are first-order linear function, they meet the requirements of convex optimization, so the optimization problem in (21) can be rewritten as follows:

$$
\begin{array}{r}
\max _{\left(W, \Sigma, x_{i}, y_{i}, \mathrm{i} \in(1,2,3,4)\right)}\left(\frac{1}{2}\left(\left(x_{1}-x_{2}-x_{3}+x_{4}\right)+\left(y_{1}-y_{2}-y_{3}+y_{4}\right)\right) \log _{2}(\mathrm{e})\right) \\
\text { St }(21 \mathrm{~b}),(23 \mathrm{a}),(23 \mathrm{~b}),(21 \mathrm{e}),(21 \mathrm{f}),(23 \mathrm{c}),(23 \mathrm{~d}),(21 \mathrm{i})
\end{array}
$$

Problem (24) is a convex optimization problem, you can use CVX ${ }^{[21]}$ toolbox, to solve the above problem.

\section{SuccessiveConvex Approximation[20]}

Letting $\tilde{x}_{2}[\mathrm{n}], \tilde{x}_{3}[\mathrm{n}], \tilde{y}_{2}[\mathrm{n}], \tilde{y}_{3}[\mathrm{n}], \tilde{\boldsymbol{W}}[\mathrm{n}], \tilde{\Sigma}[\mathrm{n}]$ be the value that $\tilde{x}_{2}, \tilde{x}_{3}, \tilde{y}_{2}, \tilde{y}_{3}, \tilde{\mathbf{W}}[\mathrm{n}], \tilde{\Sigma}[\mathrm{n}]$ iteraten times, welet $\tilde{A}, \tilde{C}, \tilde{D}, \tilde{A}_{1}, \tilde{C}_{1}, \tilde{D}_{1}$ which contains $(\tilde{\boldsymbol{W}}[\mathrm{n}], \tilde{\Sigma}[\mathrm{n}])$ to be described as $\tilde{A}[n], \tilde{C}[n], \tilde{D}[n], \tilde{A}_{1}[n], \tilde{C}_{1}[n], \tilde{D}_{1}[n]$.

Replacing (23c),(23d),(23g),(23h)with below:

$$
\begin{aligned}
& A \leq e^{\tilde{x}_{2}[n]}\left(x_{2}-\tilde{x}_{2}[n]+1\right) \\
& P_{1} e_{1}^{2} D+C B+B D \leq e^{\tilde{y}_{3}[n]}\left(x_{3}-\tilde{x}_{3}[n]+1\right) \\
& A_{1} \leq e^{\tilde{y}_{2}[n]}\left(y_{2}-\tilde{y}_{2}[n]+1\right) \\
& P_{2} e_{2}^{2} D_{1}+C_{1} B+B D_{1} \leq e^{\tilde{y}_{3}[n]}\left(y_{3}-\tilde{y}_{3}[n]+1\right)
\end{aligned}
$$

The optimization question (24)can be converted as:

$$
\max _{\left(W, \Sigma, x_{i}, y_{i}, \mathrm{i} \in(1,2,3,4)\right)}\left(\frac{1}{2}\left(\left(x_{1}-x_{2}-x_{3}+x_{4}\right)+\left(y_{1}-y_{2}-y_{3}+y_{4}\right)\right) \log _{2}(\mathrm{e})\right)
$$

S .t.

(21b), (25a), (25b),(21e), (21f), (25c), (25d), (21i), (18a)

In the continuous convex approximation, $\tilde{x}_{2}[n+1], \tilde{x}_{3}[n+1], \tilde{y}_{2}[n+1], \tilde{y}_{3}[n+1]$ is 
obtained by the previoussolution $\tilde{W}[\mathrm{n}], \tilde{\Sigma}[\mathrm{n}]$.Therefore, reaching the following expression:

$$
\begin{aligned}
& \tilde{x}_{2}[\mathrm{n}+1] \triangleq \ln (\tilde{A}[\mathrm{n}]) \\
& \tilde{x}_{3}[\mathrm{n}+1] \triangleq \ln \left(P_{1} e_{1}^{2} \tilde{D}[\mathrm{n}]+\tilde{C}[\mathrm{n}] B+B \tilde{D}[\mathrm{n}]\right) \\
& \tilde{y}_{2}[\mathrm{n}+1] \triangleq \ln \left(\tilde{A}_{1}[\mathrm{n}]\right) \\
& \tilde{y}_{3}[\mathrm{n}+1] \triangleq \ln \left(P_{1} e_{1}^{2} \tilde{D}_{1}[\mathrm{n}]+\tilde{C}_{1}[\mathrm{n}] B+B \tilde{D}_{1}[\mathrm{n}]\right)
\end{aligned}
$$

Let $\tilde{x}_{2}[\mathrm{n}+1], \tilde{x}_{3}[\mathrm{n}+1], \tilde{y}_{2}[\mathrm{n}+1], \tilde{y}_{3}[\mathrm{n}+1]$ be $x_{2}, x_{3}, y_{2}, y_{3}$ and bring them in(26), one can $\operatorname{reach} \tilde{\boldsymbol{W}}[n+1], \tilde{\Sigma}[n+1]$, then the newly obtained optimal beamforming matrix and artificial noise covariance matrix from (27). The above operation is repeated until the optimal value of the objective function converges.

According to 3.1 and 3.2 discussed above, the algorithm can be summarized as below: 1. Get a set of feasible vectors $\boldsymbol{w}$ and $z$ which can meet(26)

2. Letting $\tilde{\boldsymbol{W}}[0]=\boldsymbol{w} \boldsymbol{w}^{\mathrm{H}}, \tilde{\Sigma}[0]=z z^{\mathrm{H}}$ and settingn $=0$;

3.Loop: Put the valueinto (27) thenobtain $\tilde{x}_{2}[n+1], \quad \tilde{x}_{3}[n+1], \quad \tilde{y}_{2}[n+1], \quad \tilde{y}_{3}[n+1]$, at the same time bring it in (26) to get. At this moment, we make $n=n+1$ can get $\tilde{W}[\mathrm{n}], \tilde{\Sigma}[\mathrm{n}]$, and then bring them into the (27), continuously in accordance with the above method, until $o p(n)-o p(n-1) \leq \Delta$, at this time, the optimal beamforming matrix and AN covariance matrix can be obtained.

4. If the rank of the resulting beamforming matrix is 1 , then the decomposition can be done directly through $\tilde{W}=\left(w^{*}\right)\left(w^{*}\right)^{\mathrm{H}}$. If the rank is not 1 , an approximate solution of rank 1 can be obtained using the Gaussian random me thod mentioned in $[7,15]$.

Notice: In simulation may occur $\left(x_{1}-x_{2}-x_{3}+x_{4}\right) \leq 0$ or $\left(y_{1}-y_{2}-y_{3}+y_{4}\right) \leq 0$. The reason for this condition is that the link gain of the first or second cognitive user to the eavesdropper is much better than the link gain to other nodes, resulting in eavesdropper gaining more information than the destination node.In order to ensure the security of transmission, when secrecy rateof oneuse is negative, this node won't sends information anymoreand all the power is allocated to another user.

\section{Simulation Results and Performance Analysis}

In this section, this paperuses proposed scheme (PBNAS) to compare with the beamforming scheme (BS) [15], the null space artificial noise aided and beamforming scheme(NABS) [9] and the common artificial noise and beamforming scheme (ABS) [7]. In the simulation, the CVX toolbox is used to solve the SDP problem. Assuming that the channel is Rayleigh channel, the gain follows independent Gaussian distribution with mean 0 and variance 1 . The noise power of all nodes is $0 \mathrm{dBm}$. The remaining simulation parameters are illustrated in each simulation diagram.

Figure 2 is the relation between the number of iteration and convergencewhen we use SCA.In the simulation, the transmitting power of the two secondary nodes is fixed at $5 \mathrm{~mW}$, the total transmission power of relays is $5 \mathrm{dBm}, 15 \mathrm{dBm}, 25 \mathrm{dBm}$, the interference temperature is $0 \mathrm{dBm}\left(I_{\max }=0 \mathrm{dBm}\right)$ and the number of relays are 
7( $\left.\mathrm{N}_{\mathrm{R}}=7\right)$. This paper does 800 times independent Monte Carloexperiment. From the simulation diagram we can get that total transmit power is higher and the total security rate is also higher. After 5 times iterations, the value is stable, and through 15 iterations, the value converges. Therefore, in the following simulation, the number of iterations are set to 15 times. And the maximum value of 15 iterations is taken as the optimal value.

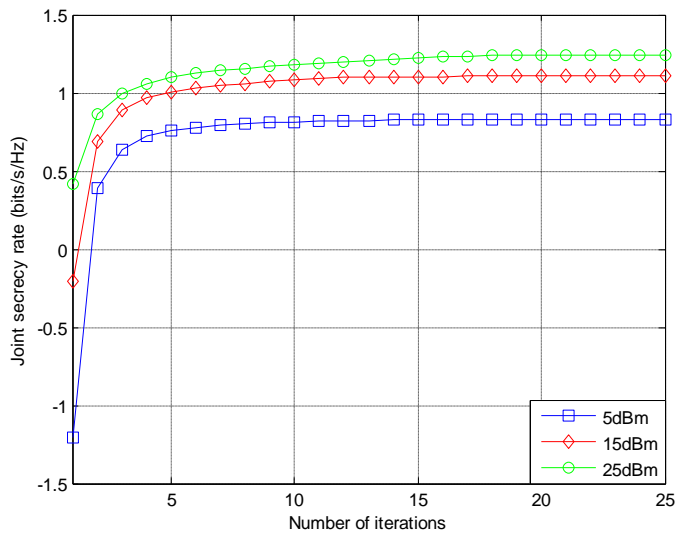

Figure 2. Secrecy rate versus number of iterations

Figure 3 showsthe relation between number of relays and secrecy ratein four transmission schemes. Set $P_{\max }=50 \mathrm{~mW}, I_{\max }=0 \mathrm{dBm}$, except the scheme of power allocation, other schemes set P1 $=\mathrm{P} 2=5 \mathrm{~mW}$. In general, with the increasingof relays' number,secrecy rate is also increasing. This is because increasing the number of relays is equivalent to increasing the number of antennas, just as increasing the gain of the array.So information destinations receive information is greater than eavesdropped. When the number of relays reaches 18, the secrecy rate will not be improved obviously. The reasonis when the relay increases to certain number,the array gain is quite obvious and the number of relay has little effect on secrecy rate. From figure 3 we can get that PBNAS has better securityperformance than other schemes.

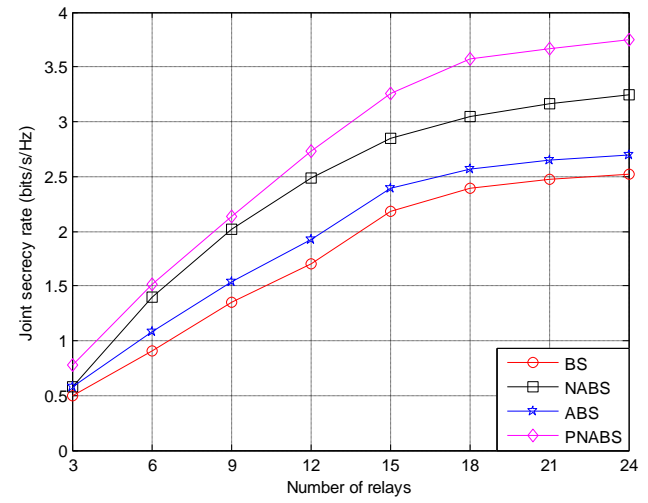

Figure 3. Secrecy rate versus number of relays

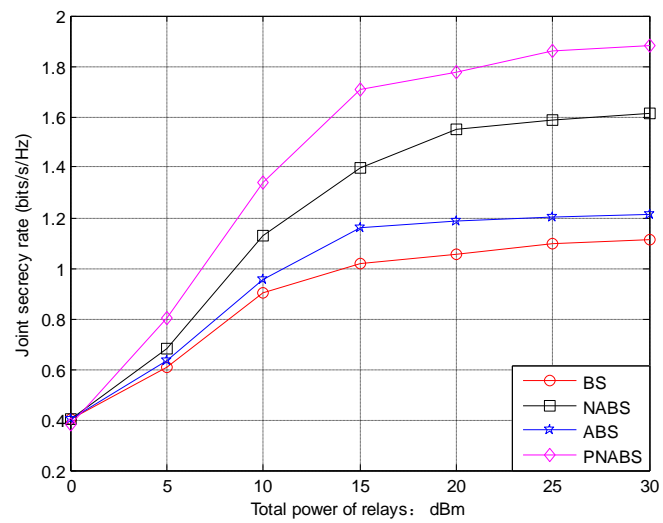

Figure 4.Secrecy rate versusdifferentpower

Figure 4 is the relation between total relay power and secrecy ratein four transmission schemes. This paper sets $\mathrm{N}_{\mathrm{R}}=7, I_{\max }=0 \mathrm{dBmin}$ the simulation. From the figure, we can see with less total power, the maximum secret rate is similar in the four schemes. However, as the total power increases, the secret rate increases too. With total power increasing relays will have enough energy to send artificial noise to interfere the eavesdropper nodes, but when the total power reaches $20 \mathrm{dBm}$, the secret rate will stabilize. In general, BS's performance is worse than the other schemes. In PNABS 
this paper adopts global search method with step of 0.01 to allocate power, the better the coefficient of the node can be allocated more power, while reducing the eavesdropping ability in the first phase. In summary, the PBNAS is best.

\section{Conclusion}

This paper researches the PLS transmission scheme in the CRN of multiple replays with two pairs of secondary nodes without direct link. A joint optimization schemewhich based on the SDP and the SCAis proposed, the original complex nonconvex problem is transformed into a convex optimization problem. Then the paper doesa global search to get optimal transmission power, at the same time optimize the artificial noise covariance and the beamforming vector to obtain the optimal secrecy rate. The results of simulation show that the PNABS scheme owns the best security performance. Next we will consider power efficiency and further improve physical layer security through the collaboration of secondary user with primary users.

\section{Acknowledge ment}

This work is supported by the Natural Science Foundation of China(61271259,61301123, 61471076), the Program for Changjiang Scholars and Innovative Research Team in University (IRT2129), the special fund of Chongqing key laboratory (CSTC), the Natural Science Foundation of Chongqing(NO. CTSC2015jcyjA40013).

\section{References}

[1] Fragkiadakis A G, Tragos E Z, Askoxylakis I G. A survey on security threats and detection techniques in cognitive radio networks[J]. IEEE Communications Surveys \& Tutorials, 2013, 15(1):428-445.

[2] Li J W, Feng Z B, Feng Z Y, et al. A survey of security issues in cognitive radio networks[J]. China Communications, 2015, 12(3): 132-150.

[3] Shu Z, Yi Q, Song C. On physical layer security for cognitive radio networks[J]. IEEE Network, 2013, 27(27):28-33.

[4] Zou Y L, Zhu J, Yang L Q, et al. Securing physical-layer communications for cognitive radio networks[J]. IEEE Communication Magazine, 2015, 53(9): 48-54.

[5] Rajesh K S and Danda B R. Advances on security threats and countermeasures for cognitive radio networks: a survey[J]. IEEE Communications Surveys \& Tutorials, 2015, 17(2):1023-1043.

[6] Chen T, Yu H, Wei G. Study on the physical layer security of cognitive radio networks and its robustness design[J]. Journal of Electronics \& Information Technology, 2012, 34(4): 770-775.In Chinese

[7] Zhang L J, Jin L, Liu L, et al. Artificial noise aided secure beamforming for multi-antenna relay systems[J]. Journal on Communications, 2014, 35(11):81-88. In Chinese

[8] Xie X Z, Xie C J. Improved transmit design for physical layer security in cognitive radio networks with multiple eavesdropper base on semi-definite 
programming[J]. Journal of Electronics \& Information Technology, 2015, 37(10):2424-2430. In Chinese

[9] Lei W J, Zuo L J, Jiang X. Physical layer security scheme resistant to multi-eavesdroppers with inaccurate channel state information in relay network[J]. Journal of Electronics \& Information Technology, 2015, 37(9):2191-2197. In Chinese

[10] Sakran H, Nasr O, Shokair M, et al. Proposed relay selection scheme for physical layer security in cognitive radio networks[J]. IET Communications, 2012, 6(16):2676-2687.

[11] He T, Zhao T. Relay selection with jamming under security constraints in cognitive radio networks[C]//International Conference on Communications and Networking in China, 2013: 63-68.

[12]Liu Y W, Wang LF, Tran T D, et al. Relay selection for security enhancement in cognitive relay networks[J]. IEEE Wireless Communications Letters, 2015, 4(1): 46-49.

[13] Mukarram A, Azzam A, Mohammed M A. Relay selection scheme for improving the physical layer security in cognitive radio networks[C]//IEEE 23nd Signal Processing and Communications Applications Conference, 2015: 495-498.

[14]Zou Y L, Benoit C, Zhu W P, et al. Relay-selection improves the security-reliability trade-off in cognitive radio systems[J]. IEEE Transactions onCommunications, 2015, 63(1): 215-228.

[15]Zhang J W, Mustafa C G. Secure relay beamforming over cognitive radio channels[C]//IEEE 45th Annual Conference on Information Sciences and Systems (CISS), $2011: 1-5$.

[16]He T, Chen H W, Liu Q. QoS-based beamforming with cooperative jamming in cognitive radio networks[C]//IEEE International Conference on Communications, Circuits and Systems (ICCCAS), 2013:42-45.

[17] Wang X, Wang K, Zhang X D. Secure relay beamforming with imperfect channel side information[J]. IEEE Transactions on Vehicular Technology, 2013, 62(5): 2140 2155 .

[18]Wang X, Zhang Z, Long K. Secure beamforming for multiple-antenna amplify-and-forward relay networks[J]. IEEE Transactions on Signal Processing, 2016, 64(6): 1477 - 1492.

[19]Luo Z Q, Ma W K, So M C, et al. Semidefinite relaxation of quadratic optimization problems[J]. IEEE Signal Processing Magazine, 2010, 27(3):20-34.

[20] Li W C, Chang T H, Lin C, et al. Coordinated beamforming for multiuser MISO Interference channel under rate outage constraints[J]. IEEE Transactions on Signal Processing, 2013, 61(5):1087-1103.

[21]Grant M C, Boyd S P. CVX: MATLAB software for disciplined convex programming, version 2.0 beta. [Online]. Available:http://cvxr.com/cvx 